\title{
Five-year risk of HIV diagnosis subsequent to I 47 hospital-based indicator diseases: a Danish nationwide population-based cohort study
}

This article was published in the following Dove Press journal:

Clinical Epidemiology

6 September 2016

Number of times this article has been viewed

\author{
Lars Haukali Omland' \\ Rebecca Legarth' \\ Magnus Glindvad Ahlström' \\ Henrik Toft Sørensen ${ }^{2}$ \\ Niels Obel' \\ 'Department of Infectious Diseases, \\ Copenhagen University Hospital, \\ Rigshospitalet, Copenhagen, \\ 2Department of Clinical Epidemiology, \\ Aarhus University Hospital, Aarhus, \\ Denmark
}

Background: It has been suggested that targeted human immunodeficiency virus (HIV) testing programs are cost-effective in populations with an HIV prevalence $>0.1 \%$. Several indicator diseases are known to be associated with increased risk of HIV infection, but estimates of HIV frequency in persons with relevant indicator diseases are nonexistent.

Methods: In a nationwide population-based cohort study encompassing all Danish residents aged 20-60 years during 1994-2013, we estimated the 5-year risk of an HIV diagnosis (FYRHD) after a first-time diagnosis of 147 prespecified potential indicator diseases. To estimate the risk of HIV diagnosis in the general population without any indicator diseases, we calculated the FYRHD starting at age 25, 35, 45, and 55 years.

Results: The risk in the male general population was substantially higher than the female general population, and the risk was lower in the older age categories. Individuals of African origin had a higher FYRHD than individuals of Danish origin. A number of diseases were identified with a FYRHD $>0.1 \%$, with infectious diseases, such as syphilis, hepatitis, and endocarditis, associated with a particularly high FYRHD. Other potential indicator diseases, such as most urologic, nephrologic, rheumatologic, and endocrine disorders were generally associated with a low FYRHD.

Conclusion: Our study identified a large number of indicator diseases associated with a FYRHD $>0.1 \%$. These data can be used as a tool for planning targeted HIV screening programs.

Keywords: HIV, indicator diseases, HIV screening, public health

\section{Introduction}

Highly active antiretroviral therapy (HAART) has substantially reduced morbidity and mortality for human immunodeficiency virus (HIV)-infected individuals, ${ }^{1}$ and the optimal timing of HAART initiation has recently been determined. ${ }^{2}$ It is important to diagnose HIV early, as immunodeficiency resulting from late presentation is associated with increased mortality ${ }^{3,4}$ and delaying HAART initiation to a very advanced stage of immunodeficiency is associated with increased mortality. ${ }^{2}$ Moreover, early diagnosis and treatment decreases the risk of disease transmission. ${ }^{5,6}$

Several HIV case-finding strategies have been proposed to enhance early diagnosis. ${ }^{7}$ While universal testing is recommended by the Center for Diseases Control and Prevention and the US Preventive Services Task Force, ${ }^{8,9}$ targeted testing was advocated by the "HIV in Europe 2007 Conference". ${ }^{10}$ It has been estimated that HIV testing in populations in whom the HIV prevalence is $0.1 \%$ or higher is cost-effective compared to interventions for other chronic conditions. ${ }^{11,12}$ A Danish case-control study identified
Correspondence: Lars Haukali Omland Department of Infectious Diseases, Copenhagen University Hospital, Rigshospitalet, DK-2100

Copenhagen $\varnothing$, Denmark

Tel +4535457726

Fax +45 35457750

Email omland@dadlnet.dk 
several diseases associated with an increased relative risk of subsequent HIV diagnosis, ${ }^{13}$ but due to its case-control design, it could not provide absolute risk estimates to guide targeted testing policies. The HIV indicator diseases across Europe study I demonstrated that patients with one of the eight preselected indicator diseases/conditions had an HIV prevalence of $>0.1 \%$, suggesting indicator disease-guided testing as a feasible way to identify occult HIV cases. ${ }^{14}$

Ideally, nationwide estimates of HIV prevalence in persons with relevant indicator diseases should be obtained to guide HIV testing policies. However, as this is not possible, other measures have to be applied. In the present study, we used the high-quality national Danish registries to estimate the 5-year risk of HIV diagnosis (FYRHD) after a diagnosis of 147 prespecified indicator diseases.

\section{Methods}

We conducted a nationwide population-based cohort study encompassing all Danish residents aged 20-60 years during the period 1994-2013. The study outcome was FYRHD after a first-time diagnosis of 147 prespecified potential indicator diseases.

\section{Setting}

Among Denmark's population of 5.5 million persons, an estimated 5,500 persons are infected with $\mathrm{HIV},{ }^{15}$ yielding a prevalence of $\sim 0.1 \%$. Denmark's tax-funded health care system provides treatment (including antiretroviral treatment) free of charge to all HIV-infected residents. During the period 1990-2012, 6,358 newly diagnosed HIV-infected patients were reported to the Danish authorities. ${ }^{16}$

\section{Data sources}

We used the unique ten-digit personal identification number assigned to all Danish residents at birth or upon immigration to avoid multiple registrations and to track individuals in national registries. We extracted data from the Danish Civil Registration System, a national registry containing information on vital status, country of birth, and dates of immigration and emigration. ${ }^{17}$ We also obtained information from the Danish National Patient Registry (DNPR) on all diagnoses made during hospital outpatient and emergency room contacts and during hospital stays in Danish nonpsychiatric hospitals. These diagnoses are coded by the attending physician according to the International Classification of Diseases, 10th revision. ${ }^{18}$ From the DNPR, we extracted the first date of HIV diagnosis and the first date of any of the indicator diseases, grouped as described in the paper by Søgaard OS et al. ${ }^{13}$

\section{Study population}

We included all Danish residents who were between the ages of 20 and 60 years at any time during the study period (January 1, 1994 to December 31, 2012) and who were born in Denmark or on the African continent. This restriction in age category was chosen to reflect the primary age of acquiring HIV diagnoses in Denmark. Patients from nonDanish Western countries and "other" countries ( $\sim 14 \%$ of the potential study population) were excluded, as they represent a heterogeneous population from countries with very different HIV prevalences.

\section{Statistical analysis}

Follow-up time was calculated from the first diagnosis date of any of the indicator diseases to the date of HIV diagnosis, emigration, death, or January 1, 2013, whichever occurred first. We computed the FYRHD and 95\% confidence intervals, considering death as a competing risk. ${ }^{19}$ For indicator diseases with no cases of HIV within 5 years of observation, we computed the upper confidence limit using the exact method based on a binomial distribution. ${ }^{20}$ Analyses were stratified according to place of birth (Denmark or Africa), sex, and age on the diagnosis date of the indicator disease at 10 -year intervals from ages 20 to 60 years. To estimate the risk of HIV diagnosis in the general population, we calculated the FYRHD starting at age 25, 35, 45, and 55 years for all strata.

SPSS version 19.0 (IBM Corporation, Armonk, NY, USA) and R software, version 2.14.2 (Vienna, Austria), were used to perform the analyses.

\section{Ethics}

According to Danish law, registry-based studies do not require approval from an ethics board. The data were anonymized by Statistics Denmark prior to analyses.

\section{Results}

We identified 4,169,600 persons who fulfilled the study inclusion criteria. Among these, 2,098,773 were males of Danish origin, 2,039,271 were females of Danish origin, and 31,556 were of African origin (both sexes). Total observation time was $>56$ million years. During follow-up, 4,162 persons were diagnosed with HIV infection, which is $79.9 \%$ of the total number of HIV diagnoses $(n=5,211)$ made in Demark during 
the study period. Characteristics of the study population are provided in Table 1.

\section{FYRHD in patient categories defined by age and origin}

The FYRHD in the Danish population at age 25, 35, 45, and 55 years is shown in Figure 1. The risk in the male general population was substantially higher than in the female general population, and the risk was lower in the older age categories. For males aged 35 years, the FYRHD was close to $0.1 \%$ (FYRHD: $0.064 \%, 95 \%$ confidence interval: $0.058 \%-0.071 \%$ ). Individuals of African origin aged 35 years had an FYRHD $>1 \%$. In general, individuals of African origin
Table I Characteristics of the study population

\begin{tabular}{llll}
\hline Characteristic & Danish & & African \\
\cline { 2 - 4 } & Male & Female & Both sexes \\
\hline Individuals & $2,098,773$ & $2,039,27 \mathrm{I}$ & $3 \mathrm{I}, 556$ \\
$\begin{array}{l}\text { Age, years } \\
\text { (median, IQR) }\end{array}$ & $32.7(20.0-47.0)$ & $33.1(20.0-47.4)$ & $26.8(20.0-33.1)$ \\
$\begin{array}{l}\text { Person-years of } \\
\text { follow-up (total) }\end{array}$ & $28,534,788$ & $27,93 \mathrm{I}, 787$ & $3 \mathrm{I} 4,210$ \\
$\begin{array}{l}\text { Person-years } \\
\text { of follow-up } \\
\text { (median, IQR) }\end{array}$ & $16.5(8.5-19.0)$ & $16.8(8.7-19.0)$ & $9.7(3.9-15.4)$ \\
$\begin{array}{l}\text { HIV cases during } \\
\text { follow-up (\%) }\end{array}$ & $2,905(0.14)$ & $510(0.03)$ & $747(2.37)$ \\
\begin{tabular}{l} 
Deaths (\%) \\
\hline
\end{tabular} & $\mathrm{II} 9,575(5.7)$ & $75,027(3.7)$ & $505(\mathrm{I} .6)$ \\
\hline
\end{tabular}

Abbreviation: IQR, interquartile range.
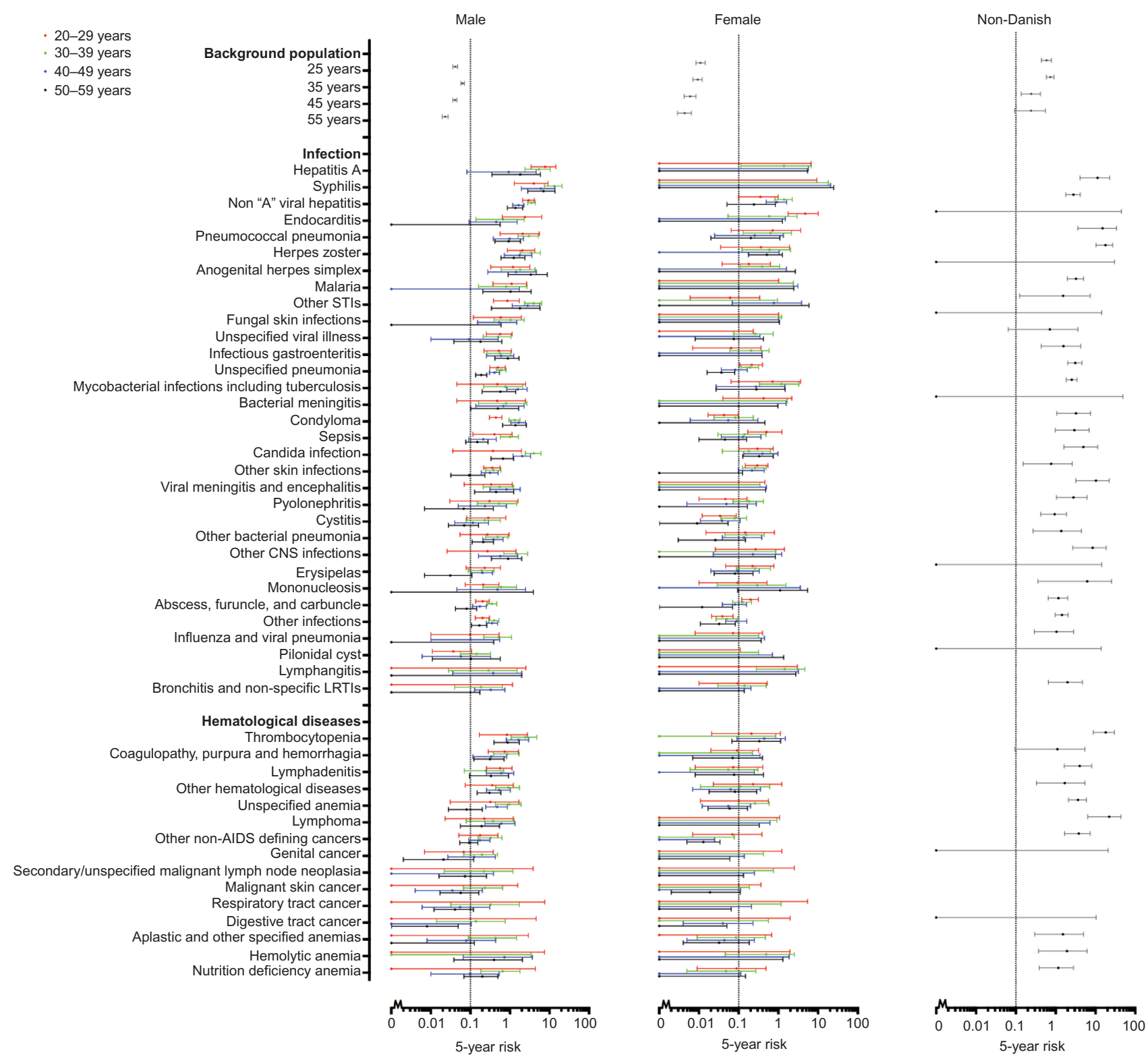

Figure I (Continued) 


$$
\begin{aligned}
& .20-29 \text { years } \\
& .30-39 \text { years } \\
& .40-49 \text { years } \\
& .50-59 \text { years }
\end{aligned}
$$

Substance abuse Substance abuse opioids Substance abuse other
Substance abuse alcohol

Poisoning

Systemic antibiotics,systemic anti-infectives and antiparasitics Antiepileptic, sedative-hypnotic and antiparkinsonism drugs

Nonopioid analgesics, Narcotics and hallucinogenics Other psychotropic drugs

$$
\text { Other substances }
$$
Alcohol

Otorhinolaryngology Peritonsillar abscess Chronic disease of tonsils and adenoids Acute tonsillitis Diseases of middle ear and mastoid Chronic pharyngitis and sinusitis Other chronic upper respiratory tract disorders Diseases of external ear Other ear, nose and throat diseases Other ear disorders Diseases of inner ear

$$
\begin{array}{r}
\text { Dermatology } \\
\text { Seborrheic dermatitis }
\end{array}
$$
Seborrheic dermatitis Papulo-squamous disorder
Other dermatitis and eczema Other dermatitis and eczema Urticaria and erythema
Other skin disease

$$
\text { Atopic dermatitis }
$$

Gastroenterology Fissure/abscess of anal and rectal regions Disease of salivary glands, oral mucosa, tongue, and lips Other gastrointestinal diseases Gastritis and duodentitis Disorder of jaws, teeth, and supporting structures Non-infective enteritis and colitis

Other diseases of intestines and peritoneum Disease of esophagus Disorder of gallbladder and biliary tract

Appendicitis

Dyspepsia

Hernia

Pancreatitis

Ophthalmology Disorder of eyelid, lacrimal system, orbit Disorders of sclera, cornea, iris, cilliary body Other eye diseases Disorders of lens -

$$
\text { ón }
$$
Other acute upper respiratory tract infections Liver diseases

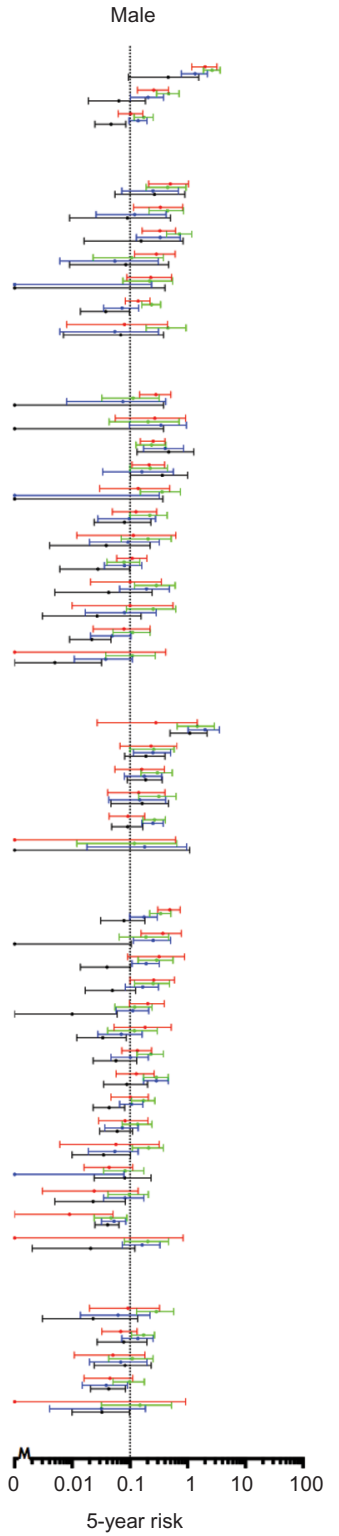

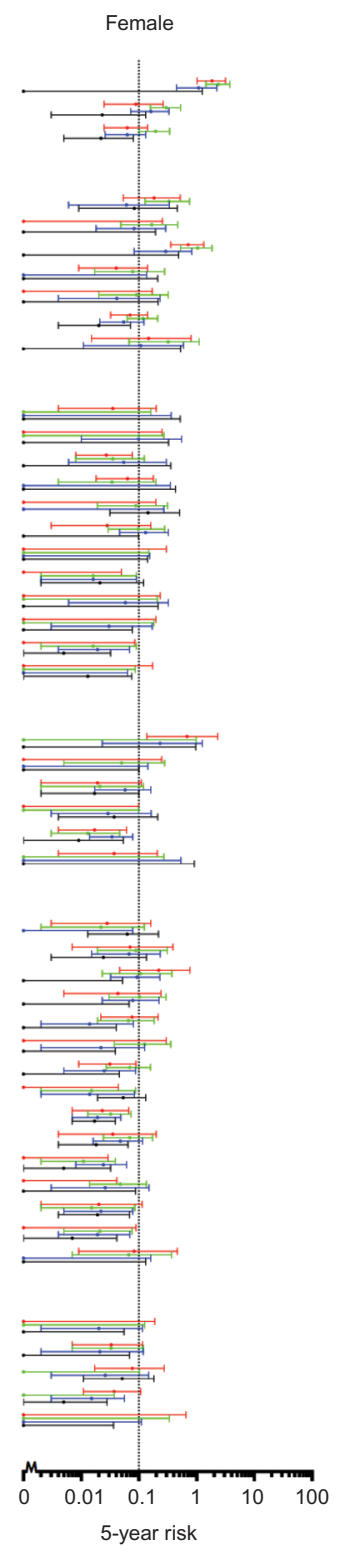

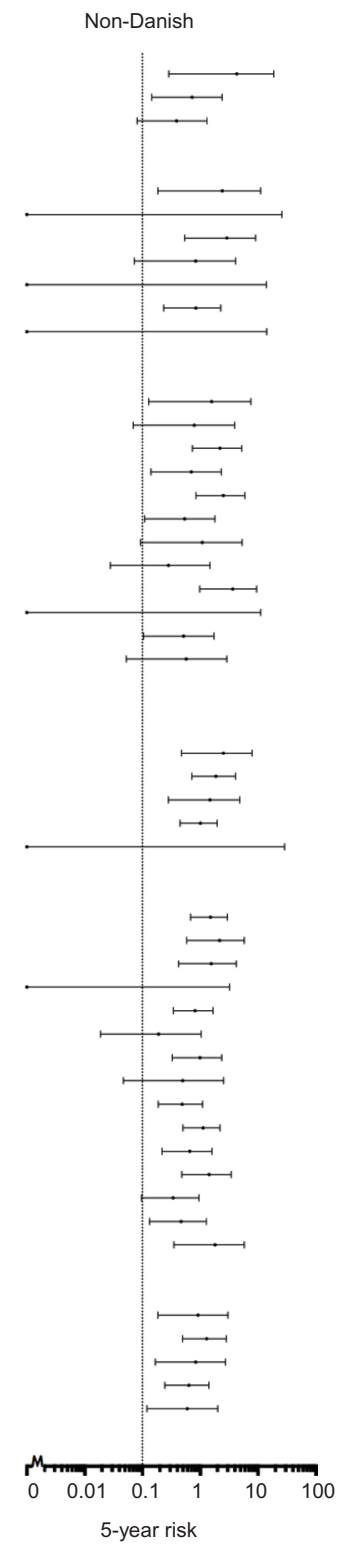

Figure I (Continued) 

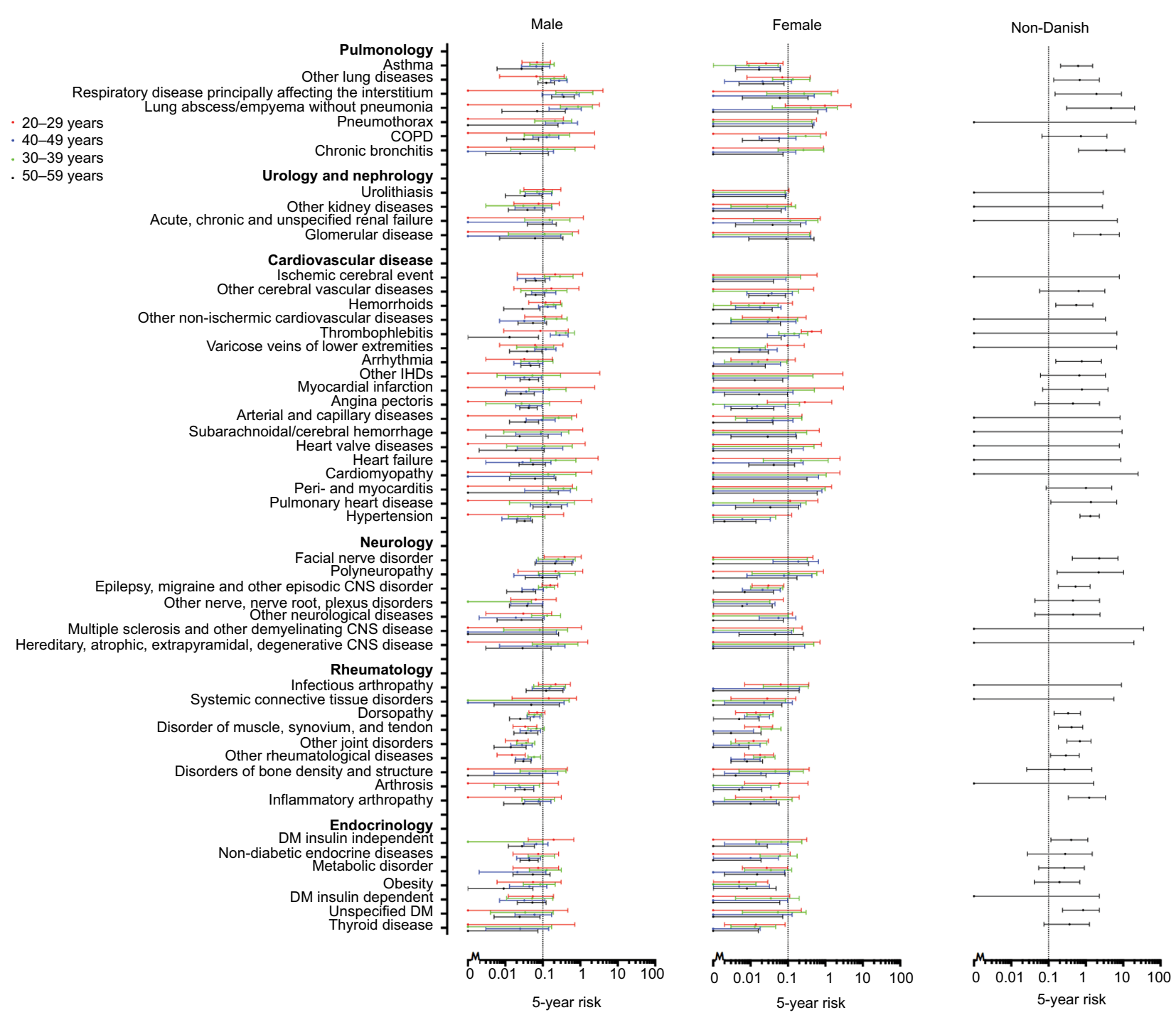

Figure I Five-year risk of human immunodeficiency virus in 147 indicator diseases for Danish males and females, and individuals of African origin. Abbreviations: CNS, central nervous system; COPD, chronic obstructive pulmonary disease; DM, diabetes mellitus; IHD, ischemic heart disease; STI, sexually transmitted infection; LRTI, lower respiratory tract infection.

had an FYRHD $>0.1 \%$. There were a total of $588(147 \times 4)$ categories defined by indicator diseases and age categories. For the Danish population, females more often than males had 0 events of HIV diagnosis in these 588 categories within the 5 years of observation. For persons of African origin, the median time from immigration to HIV diagnoses was 1.60 years (IQR: 0.34-5.34 years).

\section{FYRHD in individuals of Danish origin}

The FYRHD after a diagnosis of one of the predefined indicator diseases is illustrated in Figure 1. For both sexes, almost all the potential indicator diseases in the infectious disease category were associated with an FYRHD $>0.1 \%$ in one or more age categories.
Several hematological diseases, especially thrombocytopenia, were associated with an FYRHD $>0.1 \%$, particularly in males. Abuse of opioids was associated with an FYRHD $>0.1 \%$ in both sexes and in all age categories, while for alcohol abuse, this was the case mainly in the groups aged 30-39 and 40-49 years.

Particularly in older males, gastrointestinal diseases were associated with an FYRHD $>0.1 \%$. Among females, the only gastrointestinal diseases associated with an FYRHD $>0.1 \%$ were liver diseases.

In males, a number of skin diseases and ear, nose, and throat conditions were associated with an FYRHD $>0.1 \%$. The FYRHD was also close to or above $0.1 \%$ in males diagnosed with facial nerve disorder and neuropathy. Several 
vascular diseases also were associated with an FYRHD $>0.1 \%$ in males. Except for asthma, pulmonary diseases were associated with an FYRHD $>0.1 \%$ in one or more age and sex categories.

In some of the disease categories, such as urologic, nephrologic, rheumatologic and endocrine disorders, none or only a few indicator diseases were associated with an FYRHD $>0.1 \%$.

\section{FYRHD in individuals of African origin}

Individuals of African origin, irrespective of age category, had an FYRHD $>0.1 \%$. Furthermore, most disease categories were associated with an FYRHD $>0.1 \%$ in individuals of African origin. Possible exceptions were cardiovascular, urologic, and nephrologic diseases, for which less than half of the categories were associated with an FYRHD $>0.1 \%$. Infectious diseases were associated with an especially high FYRHD.

\section{Patient categories with a particularly high FYRHD}

The ten combinations of indicator diseases, age, and sex with the highest FYRDH among individuals of Danish origin, as well as the ten indicator diseases with the highest FYRHD among individuals of African origin, are shown in Table 1.

\section{Discussion}

In this nationwide population-based cohort study encompassing the entire Danish population over a 19-year study period, we estimated the FYRHD following a diagnosis of one of 147 prespecified indicator diseases. A number of diseases were identified with an FYRHD $>0.1 \%$, with infectious diseases associated with a particularly high FYRHD.

\section{Strengths}

Central strengths of the study are its nationwide populationbased design and long-term follow-up. To our knowledge, this study is the first to use the complete population of a nation to estimate risk of HIV diagnosis subsequent to a hospital contact for indicator diseases covering almost the whole spectrum of human diseases. The study also benefitted from the high quality of Danish registries. ${ }^{17,21-23}$

\section{Limitations}

Our study has some limitations. First, in an ideal setting, all patients should have been HIV tested at diagnosis of an indicator disease, but this was not feasible in our populationbased nationwide study. Instead, we estimated risk of an HIV diagnosis in the 5-year period following diagnosis of one of the 147 indicator diseases. Although the FYRHD could both overestimate and underestimate the true HIV prevalence, we assume that it is a good proxy for HIV infection. Any concerns arising from this approach, especially that not all patients are tested for HIV within the 5-year period and that FYRHD captures both prevalent and incident cases, are likely to be outweighed by the completeness of the population-based nationwide data used. Second, we used a hospital-based registry to identify indicator diseases. Some diseases, such as herpes zoster, ${ }^{24}$ are primarily diagnosed and treated by general practitioners. Cases leading to hospitalization are usually more severe or complicated than those diagnosed and treated exclusively by general practitioners. Therefore, our estimates are more likely to reflect the risk of HIV infection in patients diagnosed with indicator diseases at hospital admission than during a consultation with a general practitioner. Furthermore, there might be a diagnostic delay from the time of diagnosis by general practitioners and the time of diagnoses in the hospital-based registry. Third, we did not account for indicator diseases possibly appearing in clusters, as this would result in very complex statistical models that would be difficult to interpret in daily clinical practice. Fourth, it must be noted that our study is based on data from a publicly funded health care system. Denmark's hospital referral pattern might differ from that of countries with other health care systems. If the referral pattern does differ in other countries, the FYRHD associated with the 147 potential indicator diseases might also differ. Fifth, the FYRHD is highly dependent on the risk of HIV infection in the population under study, and HIV incidence is rather low in Denmark. Therefore, our results cannot readily be generalized to non-Western countries. Sixth, in the beginning of the study period, indicator diseases, to some extent, were a mixture of prevalent and incident diseases, as DNPR did not include hospital outpatient and emergency room contacts until 1995.

Finally, because FYRHD varies considerably according to age, sex, and country of origin, we stratified the analyses on these characteristics. In general, we lost statistical precision by providing estimates by population category.

\section{Possible explanations for our findings}

The increased FYRHD for some diseases, such as syphilis, condyloma, non-A hepatitis, or endocarditis, can be explained by shared routes of infection. The HIV risk associated with other diseases, such as malaria and hepatitis A, might mark immigration from or tourism to countries with a high prevalence of HIV infection. Increased susceptibility caused by 
the HIV infection and resulting immunodeficiency may lead to many of the AIDS-defining diseases. The association with HIV risk might also represent misdiagnosis of the indicator disease. For instance, Epstein-Barr virus-induced mononucleosis typically has a clinical presentation characterized by fever and lymphadenopathy, and the diagnosis is not always confirmed by virological testing. If a manifestation of acute HIV infection is diagnosed as mononucleosis, the FYRHD associated with mononucleosis will be high. Similar mechanisms likely explain the association of FYRHD with other indicator diseases, such as viral meningitis and lymphadenitis.

\section{Policy implications}

Our study has important implications for policies for HIV testing. We identified a number of indicator diseases, diagnosed in a nationwide population-based hospital system, which are associated with an FYRHD $>0.1 \%$. A $0.1 \%$ prevalence seems to be a reasonable threshold for cost-effectiveness, compared to other interventions in the health care system, and is associated with earlier HIV diagnosis. ${ }^{11,12,14}$ Infectious diseases, in particular, seem to be associated with elevated FYRHD, so one simple recommendation is to perform HIV testing on all patients hospitalized for an infectious disease. As these diseases account for $\sim 40 \%$ of all admissions to acute medical units, ${ }^{25}$ they could be the target of a focused program. The rather high FYRHD found in certain categories of the background population, especially persons of African origin and Danish males aged 35 years, suggests that it also might be cost-effective to introduce universal testing in these groups irrespective of presentation of indicator diseases.

It must be emphasized that some diseases, such as most urologic, nephrologic, rheumatologic, and endocrine disorders, had an FYRHD $<0.1 \%$. Therefore, these diagnoses should not necessarily lead to an HIV test in the absence of other risk factors. When using FYRHD as a surrogate for prevalence in deciding which categories of patients to screen for HIV, it is important to consider that the FYRHD includes both cases of unknown HIV infection at the time of indicator disease diagnosis (prevalent cases) and HIV infections occurring during the 5-year follow-up period (incident cases). Incident cases would be missed if screening is performed close to indicator disease diagnosis. Therefore, screening ideally should be repeated after 5 years to ensure identification of all cases. Furthermore, although not supported by data in our study, persons with ongoing risk-taking behavior should receive counseling about safe sex practices and regular HIV testing.

\section{Conclusion}

Our study identified a large number of indicator diseases associated with an FYRHD $>0.1 \%$. These data can be used as a tool for planning targeted HIV screening programs.

\section{Acknowledgments}

NO has received research funding from Bristol-Myers Squibb, GlaxoSmithKline, Boehringer Ingelheim and Gilead.

This work was supported by Preben og Anne Simonsens Foundation, NOVO Nordisk Foundation, Rigshospitalet, Copenhagen University, The Danish AIDS Foundation and The Augustinus Foundation.

The funding sources had no role in data collection, analysis, or interpretation; the study design; the writing of the manuscript, any aspect pertinent to the study or the decision to submit it for publication.

\section{Disclosure}

The authors report no conflicts of interest in this work.

\section{References}

1. Lohse N, Hansen A-BE, Pedersen G, et al. Survival of persons with and without HIV infection in Denmark, 1995-2005. Ann Intern Med. 2007;146(2):87-95.

2. Lundgren JD, Babiker AG, Gordin F, et al; INSIGHT START Study Group. Initiation of antiretroviral therapy in early asymptomatic HIV infection. N Engl J Med. 2015;373(9):795-807.

3. Nakagawa F, Lodwick RK, Smith CJ, et al. Projected life expectancy of people with HIV according to timing of diagnosis. AIDS. 2012;26(3):335-343.

4. Mocroft A, Lundgren JD, Sabin ML, et al. Risk factors and outcomes for late presentation for HIV-positive persons in Europe: results from the Collaboration of Observational HIV Epidemiological Research Europe Study (COHERE). PLoS Med. 2013;10(9):e1001510.

5. Marks G, Crepaz N, Janssen RS. Estimating sexual transmission of HIV from persons aware and unaware that they are infected with the virus in the USA. AIDS. 2006;20(10):1447-1450.

6. Volz EM, Ionides E, Romero-Severson EO, Brandt M-G, Mokotoff E, Koopman JS. HIV-1 transmission during early infection in men who have sex with men: a phylodynamic analysis. PLoS Med. 2013;10(12):e1001568.

7. Bayer R, Oppenheimer GM. Routine HIV screening - what counts in evidence-based policy? N Engl J Med. 2011;365(14):1265-1268.

8. Branson BM, Handsfield HH, Lampe MA, et al. Revised recommendations for HIV testing of adults, adolescents, and pregnant women in health-care settings. MMWR Recomm Rep. 2006;55(RR-14):1-17.

9. Moyer VA; U.S. Preventive Services Task Force*. Screening for HIV: U.S. Preventive Services Task Force Recommendation Statement. Ann Intern Med. 2013;159(1):51-60.

10. Gazzard B, Clumeck N, d'Arminio Monforte A, Lundgren JD. Indicator disease-guided testing for HIV - the next step for Europe? HIV Med. 2008;9 (Suppl 2):34-40.

11. Sanders GD, Bayoumi AM, Sundaram V, et al. Cost-effectiveness of screening for HIV in the era of highly active antiretroviral therapy. $N$ Engl J Med. 2005;352(6):570-585.

12. Paltiel AD, Weinstein MC, Kimmel AD, et al. Expanded screening for HIV in the United States - an analysis of cost-effectiveness. $N$ Engl J Med. 2005;352(6):586-595.

13. Søgaard OS, Lohse N, Østergaard L, et al. Morbidity and risk of subsequent diagnosis of HIV: a population based case control study identifying indicator diseases for HIV infection. PLoS One. 2012;7(3):e32538. 
14. Sullivan AK, Raben D, Reekie J, et al. Feasibility and effectiveness of indicator condition-guided testing for HIV: results from HIDES I (HIV indicator diseases across Europe study). PLoS One. 2013;8(1): e52845.

15. Sundhedsstyrelsen. Vejledning Om HIV (Human Immundefekt Virus) Og Hepatitis B Og C Virus. Available from: http://sundhedsstyrelsen. dk/publ/Publ2013/11nov/HIVHEPvej12udg.pdf. Accessed June 1, 2015.

16. Statens Serum Institut. HIV 2012. Available from: http://www.ssi.dk/ Aktuelt/Nyhedsbreve/EPI-NYT/2013/Uge\%2044\%20-\%202013.aspx. Accessed June 1, 2015.

17. Schmidt M, Pedersen L, Sørensen HT. The Danish Civil Registration System as a tool in epidemiology. Eur J Epidemiol. 2014;29(8): 541-549.

18. Lynge E, Sandegaard JL, Rebolj M. The Danish National Patient Register. Scand J Public Health. 2011;39(7 Suppl):30-33.

19. Jepsen P, Vilstrup H, Andersen PK. The clinical course of cirrhosis: The importance of multi-state models and competing risks analysis. Hepatology. 2015;62(1):292-302.
20. Jovanovic BD, Zalenski RJ. Safety evaluation and confidence intervals when the number of observed events is small or zero. Ann Emerg Med. 1997;30(3):301-306.

21. Frank L. Epidemiology. When an entire country is a cohort. Science. 2000;287(5462):2398-2399.

22. Andersen TF, Madsen M, Jørgensen J, Mellemkjoer L, Olsen JH. The Danish National Hospital Register. A valuable source of data for modern health sciences. Dan Med Bull. 1999;46(3):263-268.

23. Obel N, Reinholdt H, Omland LH, Engsig F, Sørensen HT, Hansen A-BE. Retrivability in The Danish National Hospital Registry of HIV and hepatitis B and C coinfection diagnoses of patients managed in HIV centers 1995-2004. BMC Med Res Methodol. 2008;8(1):25.

24. Pierik JGJ, Gumbs PD, Fortanier SAC, Van Steenwijk PC, Postma MJ. Epidemiological characteristics and societal burden of varicella zoster virus in the Netherlands. BMC Infect Dis. 2012;12:110.

25. Henriksen DP, Nielsen SL, Laursen CB, Hallas J, Pedersen C, Lassen AT. How well do discharge diagnoses identify hospitalised patients with community-acquired infections? - A validation study. PLoS One. 2014; 9(3):e92891.
Clinical Epidemiology

\section{Publish your work in this journal}

Clinical Epidemiology is an international, peer-reviewed, open access, online journal focusing on disease and drug epidemiology, identification of risk factors and screening procedures to develop optimal preventative initiatives and programs. Specific topics include: diagnosis, prognosis, treatment, screening, prevention, risk factor modification,

Submit your manuscript here: https://www.dovepress.com/clinical-epidemiology-journa
Dovepress

systematic reviews, risk and safety of medical interventions, epidemiology and biostatistical methods, and evaluation of guidelines, translational medicine, health policies and economic evaluations. The manuscript management system is completely online and includes a very quick and fair peer-review system, which is all easy to use. 